\title{
Top-Down and Bottom-Up Attention to Memory Are Dissociated in Posterior Parietal Cortex: Neuroimaging and Neuropsychological Evidence
}

\author{
Elisa Ciaramelli, ${ }^{1}$ Cheryl Grady, ${ }^{1,2}$ Brian Levine, ${ }^{1,2,3}$ Jon Ween, ${ }^{4}$ and Morris Moscovitch ${ }^{1,2,5}$ \\ ${ }^{1}$ Rotman Research Institute, Baycrest, Toronto, Ontario M6A 2E1, Canada, ${ }^{2}$ Department of Psychology, University of Toronto, Ontario M5S 3G3, Canada, \\ ${ }^{3}$ Department of Medicine (Neurology), University of Toronto, Toronto, Ontario M5S 1A8, Canada, and ${ }^{4}$ Kunin-Lunenfeld Applied Research Unit and \\ ${ }^{5}$ Department of Psychology, Baycrest, Toronto, Ontario M6A 2E1, Canada
}

\begin{abstract}
Posterior parietal cortex is active during episodic memory retrieval, yet its role remains unclear. According to a recent proposal, dorsal parietal cortex (DPC) allocates top-down attention to memory retrieval, whereas ventral parietal cortex (VPC) mediates the bottom-up attentional capture by retrieved contents, i.e., the Attention-to-Memory (AtoM) hypothesis. Here, for the first time, functional magnetic resonance imaging (fMRI) and lesion techniques were combined in a single study to test the role of parietal cortex in episodic retrieval. Participants studied word pairs and then detected studied (target) words among new words. In some conditions, a studied word cued the upcoming target word, facilitating recognition performance. In line with the AtoM hypothesis, left DPC was engaged when participants searched for/anticipated memory targets upon presentation of relevant memory cues and predicted the ensuing behavioral advantage. In contrast, left VPC predicted efficacy and speed of target detection on noncued trials and was largest for memory targets that were invalidly cued. Consistent with fMRI evidence, patients with lesions in DPC did not benefit from memory cueing, whereas patients with lesions in VPC had problems recognizing unexpected memory targets. These results support the AtoM hypothesis that DPC and VPC mediate top-down and bottom-up attention to memory retrieval, respectively.
\end{abstract}

\section{Introduction}

Recent neuroimaging studies (Wagner et al., 2005; Cabeza, 2008; Ciaramelli et al., 2008; Vilberg and Rugg, 2008) have shown that posterior parietal cortex (PPC) is more active for correctly recognized items than for correctly rejected items, suggesting PPC is implicated in episodic retrieval. PPC, however, is not conventionally associated with memory, primarily because patients with lesions in PPC do not show dramatic memory problems (Luria, 1976). Whether PPC has a crucial role during retrieval and what this role might be are still under debate.

The PPC is known to support attentional processes (Posner and Petersen, 1990) and also might provide "attentional support" to memory retrieval (Wagner et al., 2005). One proposal is the Attention-to-Memory (AtoM) hypothesis (Cabeza et al., 2008; Ciaramelli et al., 2008). The AtoM hypothesis makes a distinction between the mnemonic roles of dorsal parietal cortex (DPC) and ventral parietal cortex (VPC) based on the differential roles these regions play in attention. The DPC is thought to mediate topdown attention, which enables selection of stimuli based on internal goals, whereas VPC mediates bottom-up attention, which

Received March 12, 2009; revised Jan. 31, 2010; accepted Feb. 23, 2010.

This research was supported by a Marie Curie individual fellowship to E.C. and by a Canadian Institutes of Health Research grant to M.M. and C.G. We thank Roberto Cabeza, Giuseppe di Pellegrino, Elisabetta Làdavas, and Nicole Lazar for helpful comments.

Correspondence should be addressed to Elisa Ciaramelli, Rotman Research Institute, 3560 Bathurst Street, Toronto, 0N M6A 2E1, Canada. E-mail: eciaramelli@rotman-baycrest.on.ca.

DOI:10.1523/JNEUROSCI.1209-09.2010

Copyright $\odot 2010$ the authors $\quad 0270-6474 / 10 / 304943-14 \$ 15.00 / 0$ enables detection of relevant stimuli (Marois et al., 2000; Corbetta and Shulman, 2002). Consistently, in a "Posner" paradigm, DPC was maximally engaged during the cue period, when participants searched for a target, whereas VPC was exclusively engaged during target detection (Corbetta et al., 2000). Moreover, regions in VPC were found to respond more strongly to targets that were invalidly compared with validly cued, consistent with the view that this region mediates reorienting of attention toward unexpected targets (Corbetta et al., 2000). When an important stimulus appears outside the current focus of attention, VPC may function as a switch or resetting mechanism that promotes a shift in the locus of attention, thus enabling detection of relevant (yet previously unattended) stimulation (Corbetta et al., 2008, Shulman et al., 2009). Indeed, patients with hemispatial neglect following right VPC lesions are disproportionately impaired at detecting contralesional targets after invalid cueing (Friedrich et al., 1998).

According to the AtoM hypothesis, DPC and VPC serve roles in memory retrieval analogous to those they play in attention: DPC would mediate the allocation of attentional resources to memory retrieval (top-down AtoM). Processing in DPC, therefore, is expected to be prominent when retrieval relies on controlled operations, such as memory search. VPC, on the other hand, would mediate the bottom-up capture of attention by retrieved contents. Processing in VPC, therefore, is expected to be maximal when memory contents are retrieved spontaneously and rapidly. Although recent reviews of recognition memory studies have provided support for the AtoM hypothesis by show- 
ing that effects related to top-down and bottom-up AtoM tend to localize in DPC and VPC, respectively (Cabeza et al., 2008; Ciaramelli et al., 2008; Hutchinson et al., 2009), no study has provided experimental data differentiating the neural activity related to top-down and bottom-up AtoM within the same paradigm.

We conducted a functional magnetic resonance imaging (fMRI) and a neuropsychological study using a "Posner-like" recognition paradigm. After studying word pairs, participants classified single words as studied or new. To-be-recognized words could be "cued" by an old word, a new word, or a meaningless stimulus. Most old word cues preceded the word with which they were paired at study, facilitating recognition. Appearance of an old word cue, therefore, encouraged participants to search for its original associate to anticipate the upcoming memory target. If DPC is related to top-down AtoM, then DPC activity should be stronger when participants searched for/anticipated upcoming targets upon presentation of relevant cues than when no cue was provided. Moreover, patients with lesions in DPC should not benefit from valid memory cueing. On the other hand, if VPC is related to bottom-up AtoM, then VPC activity should predict recognition accuracy and speed on noncued trials and be larger for memory targets that were invalidly compared with validly cued. Moreover, patients with lesions in VPC should have problems detecting memory targets, especially if invalidly cued.

\section{Materials and Methods}

\section{fMRI study}

Participants. Fourteen young adults (10 females; age range 20-33, mean age 26 years) participated in the fMRI study. All participants were healthy, right-handed, native English speakers with no psychiatric or neurological history. They gave informed consent for the study, which was approved by the ethics committee of the Rotman Research Institute (Toronto, Canada).

Stimuli and procedure. Four hundred and eighty words (mean frequency $=36.6, \mathrm{SD}=34.9$ ), between four and eight letters long, were selected from the Kucera and Francis (1967) pool. Words were randomly assigned to one of the eight runs composing the experimental paradigm. Within each run, 42 of the 60 words were selected randomly to form the study pairs. The other 18 words were not studied but served as distracters or nonstudied cues in the test phase.

The task consisted of eight runs. Each run involved a study phase and a cued recognition phase. At study, participants viewed 21 word pairs and, for each pair, were instructed to form a sentence that included both words. Each pair was presented for $3 \mathrm{~s}$ followed by a fixation cross, which was presented for $500 \mathrm{~ms}$. A cued recognition memory phase, which consisted of 30 trials, followed immediately afterward. The beginning of each trial was signaled by a fixation cross that stayed in the center of the screen for $500 \mathrm{~ms}$. A cue stimulus was then presented in the center of the screen for $1900 \mathrm{~ms}$. The cue stimulus could be a studied word (i.e., the word on the left of a studied pair), a new word, or a meaningless stimulus (i.e.,@@@@).After a 100 ms delay, a target word appeared in the middle of the screen for $500 \mathrm{~ms}$. The target word could be a studied word (i.e., the word on the right of a studied pair) or a new word.

Participants were instructed to maintain attention in the middle of the screen, where the cue stimulus appeared, wait for the appearance of the target word, and then decide, as quickly and as accurately as possible, whether the target word was old or new. It was emphasized to subjects that they had to respond to the episodic status of the target word only, although consideration of the cue stimulus could be beneficial to performance. Subjects responded by pressing one of two keys, located on an MRI-compatible response pad, with their right (dominant) hand according to whether they judged the target word to be old or new. The $2 \mathrm{~s}$ interval between appearance of the cue and presentation of the target was chosen because testing with another cohort using the same material revealed that subjects need $\sim 2 \mathrm{~s}$ to recall the original associate of a cue.
Therefore, a cue-target interval of $2 \mathrm{~s}$ maximized the duration of memory search per trial while assuring that brain activity during the cue phase was mainly related to memory search/anticipation of the upcoming target and not to the maintenance of mnemonic expectations in working memory until presentation of the target. An intertrial interval (ITI) ranging from 0 to $4 \mathrm{~s}$ was interspersed across test trials to "jitter" the onset times of trials and allow for event-related fMRI analyses. Figure 1 illustrates the experimental paradigm.

As shown in Figure 1, the experimental paradigm involved seven types of trial determined by the nature of the target and the cue. In four types of trial, the target was a studied word. A studied target word could be preceded by the following: (1) an old word, the one with which the target was paired at study (i.e., "Intact" trial; six trials per run); (2) an old word, one that was not paired with the target word at study (i.e., "Recombined" trial; three trials per run); (3) a new word (i.e., "NewOld" trial; six trials per run); or (4) a meaningless stimulus (i.e., "NoOld" trial; six trials per run). In the remaining three types of trial, the target was a new word. A new target word could be preceded by the following: (1) a studied word (i.e., "OldNew" trial; three trials per run); (2) a new word (i.e., "NewNew" trial; three trials per run); or (3) a meaningless stimulus (i.e., "NoNew" trial; three trials per run).

We expected that when the cue was an old word, participants would search for its original associate to anticipate the upcoming target. Retrieving that cue word A was studied with word B would make it relatively easy to decide that $B$ is a studied word when it is presented as the target stimulus (i.e., Intact condition). To encourage participants to initiate retrieval attempts upon presentation of old word cues, these were made valid. That is, the frequency with which old word cues preceded their original associate (Intact condition) compared with another studied word (Recombined condition) was 2:1. In contrast, no search activity should be triggered by meaningless cues or by new word cues that, because of their low familiarity in the experimental context, were not perceived as useful links to the study phase. In the Recombined condition, memory search is engaged but the resulting advance information is not useful for the recognition task. Thus, participants should be more efficient at recognizing studied words in the Intact compared with the NoOld, NewOld, and Recombined conditions.

fMRI data acquisition. Images were acquired at Baycrest Hospital (Toronto, Canada) on a 3 tesla Siemens Magnetom Trio whole-body scanner with a matrix 12 channel head coil. Functional volumes were obtained using a whole-head $T_{2}{ }^{*}$-weighted echo-planar image (EPI) sequence [repetition time $\left(T_{\mathrm{R}}\right): 2 \mathrm{~s}$; echo time $\left(T_{\mathrm{E}}\right): 30 \mathrm{~ms}$; flip angle: $70^{\circ} ; 28$ oblique axial slices with interleaved acquisition; $3.1 \times 3.1 \times 5 \mathrm{~mm}$ voxel resolution; field of view (FOV): $20 \mathrm{~cm}$; acquisition matrix: $64 \times 64$ ]. Physiological data (heart and respiration rates) were acquired during the scanning session. Anatomical images were acquired using a MP-RAGE sequence $\left(T_{\mathrm{R}}: 2 \mathrm{~s} ; T_{\mathrm{E}}: 2.63 \mathrm{~s} ; 160\right.$ oblique axial slices with a $1 \mathrm{~mm}^{3}$ voxel size; FOV: $25.6 \mathrm{~cm}$; acquisition matrix: $256 \times 256$ ).

fMRI data analysis. Although scanning took place during both encoding and retrieval, the present study focuses on fMRI data collected at retrieval. The fMRI data were processed using the Analysis of Functional NeuroImages (AFNI) software (Cox, 1996). The initial five time points from each image volume were removed from analyses to allow for the brain magnetization to stabilize. EPI time series data were corrected for cardiac and respiratory parameters (program 3dretroicor). Time series data were spatially coregistered (program 3 dvolreg) to correct for small head motion using a three-dimensional Fourier transform interpolation. Each run was then normalized based on the mean intensity of the signal. Individual analysis was performed by generating the hemodynamic response function model for each condition based on the convolution of the time points beginning with the fixation stimulus that began each trial, using a gamma function (Cox, 1996). For each subject, maps of brain activity for the seven trial types of interest (Intact, Recombined, NoOld, NewOld, OldNew, NoNew, NewNew), which were modeled as unique events (cue phase plus target phase), were generated by fitting a general linear model to the measured fMRI time series at each voxel (program $3 \mathrm{dDeconvolve).} \mathrm{Only} \mathrm{correct} \mathrm{trials} \mathrm{were} \mathrm{included} \mathrm{in} \mathrm{the} \mathrm{analyses.}$

An additional model was estimated using a regression analysis to test for brain areas that correlated with response times (RTs) in different 


\section{A Study phase}

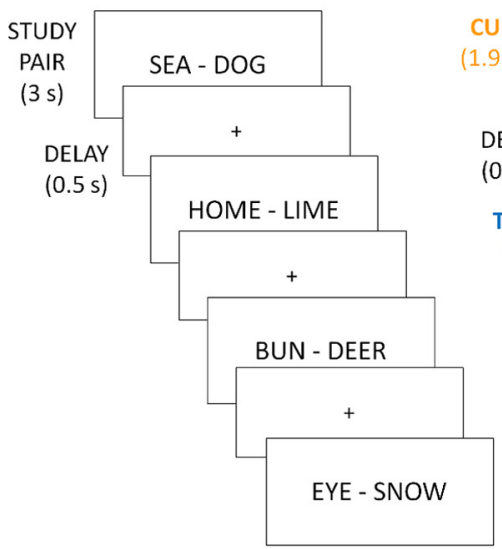

B Test trial types

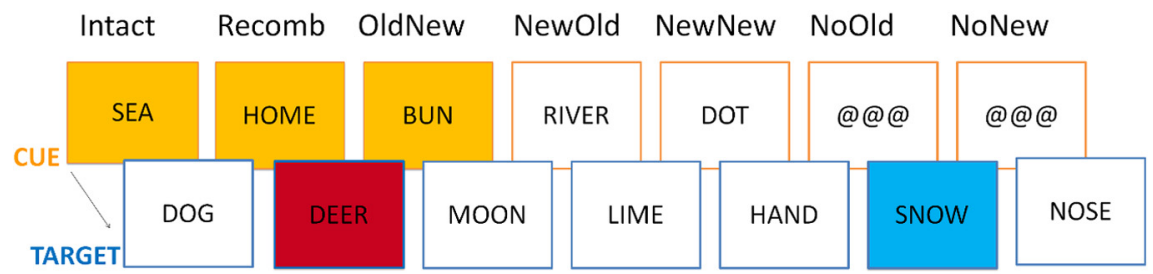

Top-down AtoM

Bottom-up AtoM

- Memory Detection

- Reorienting to Memory

Figure 1. Schematic representation of the experimental paradigm. $\boldsymbol{A}$, Participants studied word pair, and then underwent a cued recognition memory task (scanned). A cue stimulus was presented followed by a delay period, and then a target word appeared and participants decided whether the target word had been studied or not. $\boldsymbol{B}$, There were seven types of test trials, determined by the nature of the cue and the target. The cue stimulus could be a studied word, a new word, or a meaningless stimulus (i.e.,@@@).Targetwords could be studied or new.In Intact trials, the cue was a studied word and the target was the word that was paired with it at study. In Recombined (Recomb) trials, both the cue and the target were studied words but belonged to different study pairs. In OldNew trials, the cue was a studied word and the target was a new word. In NewOld trials, the cue was a new word and the target was a studied word. In NewNew trials, the cue was a new word and the target was a new word. In Noold trials, the cue was a meaningless stimulus and the target was a studied word. In NoNew trials, the cue was a meaningless stimulus and the target was a new word. Top-down AtoM was engaged when the cue was a studied word, and therefore individuals searched for/anticipated the upcoming memory target (Intact, Recombined, OldNew conditions). Bottom-up AtoM was engaged when participants detected memory targets in the absence of cues (i.e., Memory Detection; NoOld condition) and when participants detected memory targets that were invalidly cued (i.e., Reorienting to Memory; Recombined condition). See Materials and Methods for a more detailed explanation of procedures.

conditions. The model was the same as that described above, except that an additional condition-specific regressor was added. This regressor consisted of the RT for each trial converted to a $Z$ score based on the mean RT per condition for each subject. Before group analysis, the activation maps for each participant and each condition were spatially normalized to an average volume of 152 normal skull-stripped brains (www.bic.mni.mcgill. ca) matching a Talairach template (Talairach and Tournoux, 1988). Datasets were then resampled with a $2 \times 2 \times 2$ voxel dimension (program @auto_tlrc) and spatially smoothed with an $8 \mathrm{~mm}$, full-width, half-maximum Gaussian kernel (program 3dmerge). Group level analysis was performed with repeated-measures ANOVA and post hoc twotailed $t$ statistics to examine the relevant contrasts.

Unless otherwise specified, individual contrasts were assessed using an uncorrected threshold of $p<0.001$, with a cluster extent of $>15$ contiguous voxels. For analyses investigating regional overlap across different contrasts, a region was considered reliable at a conjoint significance of $p<0.001$ (see below), according to Fisher's method of estimating the conjoint significance of independent tests (Fisher, 1950; Lazar et al.,
2002). Exclusive masking was used to identify voxels for which effects were not shared between two contrasts. The individual contrasts used as exclusive masks were thresholded at $p<0.15$. Note that the more liberal the threshold of an exclusive mask, the more conservative the masking procedure.

When we held an a priori hypothesis about the localization of a predicted effect, we corrected for multiple comparisons using a smallvolume correction (Kuhl et al., 2007; Uncapher and Rugg, 2009). Because retrieval success effects were expected to involve both DPC and VPC (Ciaramelli et al., 2008), parietal regions involved in retrieval success were smallvolume corrected using an anatomical mask of left PPC [i.e., Brodmann areas (BAs) 7, 19, 39, and 40]. For DPC regions involved in topdown AtoM, we created a region of interest (ROI) centered on the median coordinates of activations related to top-down AtoM effects from a previous review $(x=-36, y=-57, z=$ 42) (Ciaramelli et al., 2008), with radius of 15 $\mathrm{mm}$. Analogously, for VPC regions involved in bottom-up AtoM, we created an ROI centered on the median coordinates of activations related to bottom-up AtoM effects (high memory confidence) from a previous review $(x=$ $-48, y=-63, z=25$ ) (Cabeza et al., 2008), with radius of $15 \mathrm{~mm}$. With the voxelwise threshold $p<0.001$, the mapwise false positive rate for all reported PPC regions, estimated using a Monte Carlo procedure (as implemented in the AlphaSim program in the AFNI software package), was $p<0.05$.

Three sets of analyses were performed to investigate retrieval success effects, effects related to top-down AtoM, and effects related to bottom-up AtoM. All our analyses involved whole trials (i.e., cue phase plus target phase).

Overall retrieval success. To begin, we sought to replicate previous results regarding the brain regions mediating retrieval success, i.e., correct recognition of studied items versus correct rejection of unstudied items. We compared brain activity related to hits (collapsed across the Intact, Recombined, NoOld, and NewOld conditions) with that related to correct rejections (collapsed across the OldNew, NoNew, and NewNew conditions).

Top-down AtoM. To identify the brain regions associated with top-down AtoM, which would be reflected in cue-related activity, we compared activity across trials that differed in the cue phase. Thus, we compared conditions in which subjects were cued by an old word (i.e., Intact, Recombined, and OldNew conditions) and therefore could search for/anticipate the upcoming target with conditions in which they were not cued or cued by a new word (NoOld, NewOld, NoNew, NewNew conditions). We performed a conjunction analysis looking for regions that were active in the contrasts (Intact - NoOld) AND (Recombined - NoOld) AND (OldNew - NoNew) while excluding those active in the contrasts (NewOld - NoOld) AND (NewNew - NoNew). The individual contrasts used as inclusive masks were thresholded at $p<0.01$. Because two of the inclusive masks were linearly independent, the conjoint probability associated with the conjunction can be estimated at $p<0.001$ (Fisher, 1950; Lazar et al., 2002).

By requiring top-down AtoM regions to be simultaneously active in the contrasts (Intact - NoOld), (Recombined - NoOld), and (OldNew NoNew), we ensured that activity was related to search processes triggered 
by relevant memory cues present across the three contrasts and not by effects related to a specific contrast, such as context reinstatement (e.g., Intact - NoOld) or violation of mnemonic expectations (e.g., Recombined - NoOld). On the other hand, by excluding activity associated with new cues, we ensured that the cue-related activation was not merely related to the evaluation of word versus nonword stimuli.

Bottom-up AtoM. To identify the brain regions associated with bottom-up AtoM, which would be seen in target-related activity, we compared activity across trials that differed in the target phase. In perception, regions associated with bottom-up attention have the following two properties: 1) they are active during target detection (as opposed to cue presentation), and 2) they respond more strongly to targets that were invalidly compared with validly cued (Corbetta et al., 2000). Thus, to reveal the brain regions associated with bottom-up AtoM, we performed two sets of analyses, the first looking for regions that were related to detection of memory targets when no cue was provided (i.e., Memory Detection), the second looking for regions related to Memory Detection that responded more strongly to unexpected than to expected memory targets (i.e., Reorienting to Memory).

Memory detection. To reveal the brain regions responsive to detection of memory targets, we focused on trials in which no cue was provided and compared trials in which the target was present (NoOld condition) with those in which it was not (NoNew condition).

Reorienting to Memory. To reveal the brain regions mediating detection of unexpected memory targets, we looked for brain regions related to Memory Detection that exhibited more activity for memories that were invalidly cued, i.e., cued by a word that was studied in association with a different word (Recombined condition), compared with memories that were validly cued, i.e., cued by their original associate (Intact condition). Thus, we performed a conjunction analysis looking for regions that were active in the contrasts (NoOld - NoNew) AND (Recombined - Intact). The (NoOld - NoNew) contrast was thresholded at $p<0.002$, and the (Recombined - Intact) contrast was thresholded at $p<0.05$, resulting in a conjoint probability of $p<$ 0.001 (Fisher, 1950; Lazar et al., 2002).

However, because in the Recombined condition subjects viewed words that belonged to two studied pairs (as opposed to just one in the Intact condition), in this condition they could potentially recall two studied pairs as opposed to one. As a result, the (Recombined - Intact) contrast, designed to index detection of memory targets that violate mnemonic expectations, might also index the amount of information retrieved. To avoid this potential confound, we exclusively masked the conjunction of (NoOld - NoNew) AND (Recombined - Intact) contrasts with the contrast (Recombined - OldNew). Because both the Recombined and the OldNew condition entail violation of mnemonic expectations, but only the Recombined condition offers the possibility to recall two studied pairs, the (Recombined - OldNew) contrast contains voxels associated with expectation violation that were also sensitive to the amount of information retrieved. By excluding those voxels from our conjunction, we ensured that regions associated with Reorienting to Memory indexed detection of unexpected memory targets and not the additional retrieval possibly associated with consideration of these events.

In addition to AFNI-based contrasts, follow-up ROI analyses were performed for certain significant clusters in the group contrast analyses. From each subject and ROI, the mean percentage signal change across all significant voxels was extracted for each of the seven conditions. These values were entered into repeated-measures ANOVAs and used to perform correlations between brain activity and relevant behavioral indices.

\section{Neuropsychological study}

To provide converging evidence in support of the AtoM hypothesis, we tested patients with lesions centered on the VPC or the DPC using the same paradigm used in the fMRI study. Previous reviews of recognition memory studies have shown that, although activity related to top-down and bottom-up AtoM was more consistent in the left PPC, it was also present in the right PPC (Ciaramelli et al., 2008). For this reason, for comparison purposes both left- and right-lesioned patients were included in the study.
Participants. Eight individuals with damage in PPC and eight agematched healthy individuals participated in the study. Seven patients were recruited at Baycrest Hospital, Toronto, Canada, and one patient was recruited at the Center for Studies and Research in Cognitive Neuroscience, Cesena, Italy (supplemental Table 1s, available at www. jneurosci.org as supplemental material). Patients were selected on the basis of the location of their lesion evident on MRI or computed tomography $(\mathrm{CT})$ scans. Included patients had a lesion in PPC, which in no case had invaded the medial or limbic region, and no other diagnosis likely to affect cognition or interfere with the participation in the study (e.g., psychiatric or cerebrovascular disease, alcohol abuse). All patients were in the stable phase of recovery (at least 12 months postmorbid).

The area of damage was determined by visual inspection on axial view by a radiologist. For a lesion to be traced, it had to appear in more than one slice with a diameter of at least $3 \mathrm{~mm}$ on one of the slices. The boundary of the lesion was traced from MRI or CT images on a standardized brain using the software MRIcro (Rorden and Brett, 2000). Figure 2 shows the extent of damage for each patient. Patients were divided into two groups depending on the location of brain damage within PPC. A group of patients had lesions centered in VPC that did not extend to the DPC (VPC patients; $N=4$ ). Two patients had left lesions, and two patients had right lesions. VPC patients had a mean age of 62 years (range 47-74) and a mean education of 12 years (range 8-14). The other group of patients had lesions in DPC (DPC patients; $N=4)$. Three patients had left lesions, and one patient had right lesions. In one case (patient 1051), the lesion extended into the VPC. We decided to include this patient in the DPC group, because a lesion in DPC was expected to cause deficits in top-down AtoM regardless of the status of VPC. We report on the performance of patient 1051 separately in Results. DPC patients had a mean age of 58 years (range 47-75) and a mean education of 15 years (range 12-17). Each patient was matched to a healthy individual on the basis of age and education. Healthy controls had a mean age of 60 years (range 47-75) and a mean education of 13 years (range 10-17).

Neuropsychological assessment. Patients underwent a neuropsychological battery assessing general cognitive functioning, vocabulary, hemispatial neglect, executive function, long-term memory, and working memory (supplemental material, available at www.jneurosci.org). Overall, patients were within the normal range on general cognitive functioning and vocabulary. Patients did not show symptoms of spatial neglect. A few patients, two from the DPC group (patients 1051 and AS) and one from the VPC group (patient 1022), were impaired on the executive measures. They showed increased response times in part B of the Trail Making Test compared with healthy controls. Patient 1022 was also impaired on the Wisconsin Card Sorting Test. On the verbal memory tasks, overall the patients scored below average, although only two patients, one from the DPC group and one from the VPC group, showed pathological scores. Working memory was intact in all the patients except for 1022 (for individual results, supplemental Table 2s, available at www. jneurosci.org as supplemental material).

Stimuli and procedure. The materials and the experimental paradigm were the same as in the fMRI study, with one exception. In the test phase, the cue stimulus was presented for $1 \mathrm{~s}$ and, after a $1 \mathrm{~s}$ delay, the target word appeared for $1 \mathrm{~s}$. The ITI was $1500 \mathrm{~ms}$. This paradigm made it easier for patients to distinguish the cue and the target and allowed them more time to evaluate the target. A pilot study using this paradigm yielded results similar to those obtained in the fMRI study. To reduce fatigue, patients completed a limited number of runs, ranging from 4 to 6 . There was no significant difference in the number of runs completed by VPC and DPC patients ( 5.25 vs $5 ; p=0.50$ ). Each normal control completed the same number of runs as the patient with whom she or he was paired. English words were replaced by Italian words of comparable frequency (Barca et al., 2002) for testing Italian patient S.M.

\section{Results \\ fMRI study}

Behavioral results

Figure 3 shows accuracy and RTs for memory decisions on old and new words across conditions. An ANOVA on the proportion of hits with condition (Intact, Recombined, NewOld, NoOld) as 


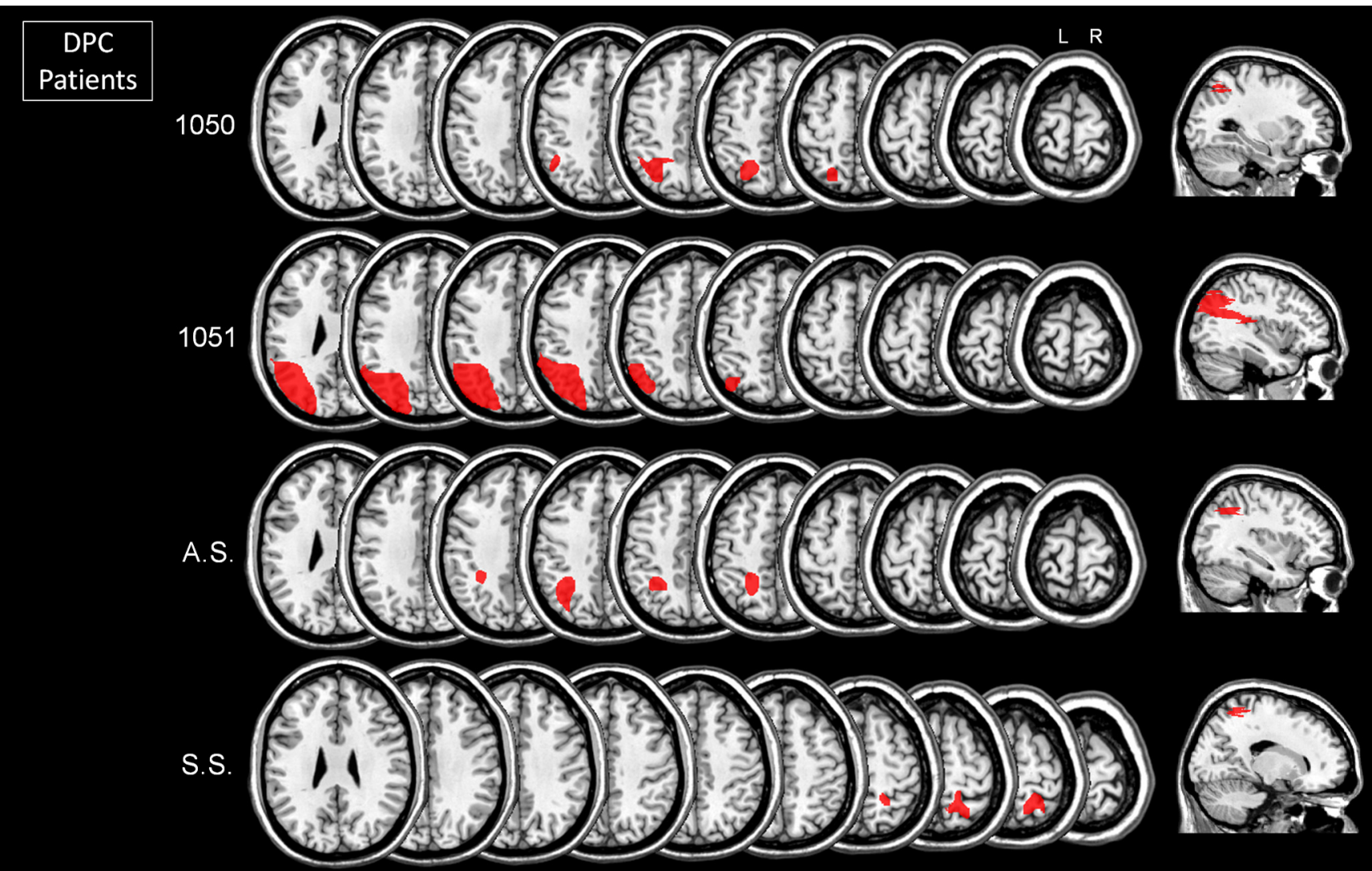

\begin{tabular}{|c|}
\hline VPC \\
Patients
\end{tabular}
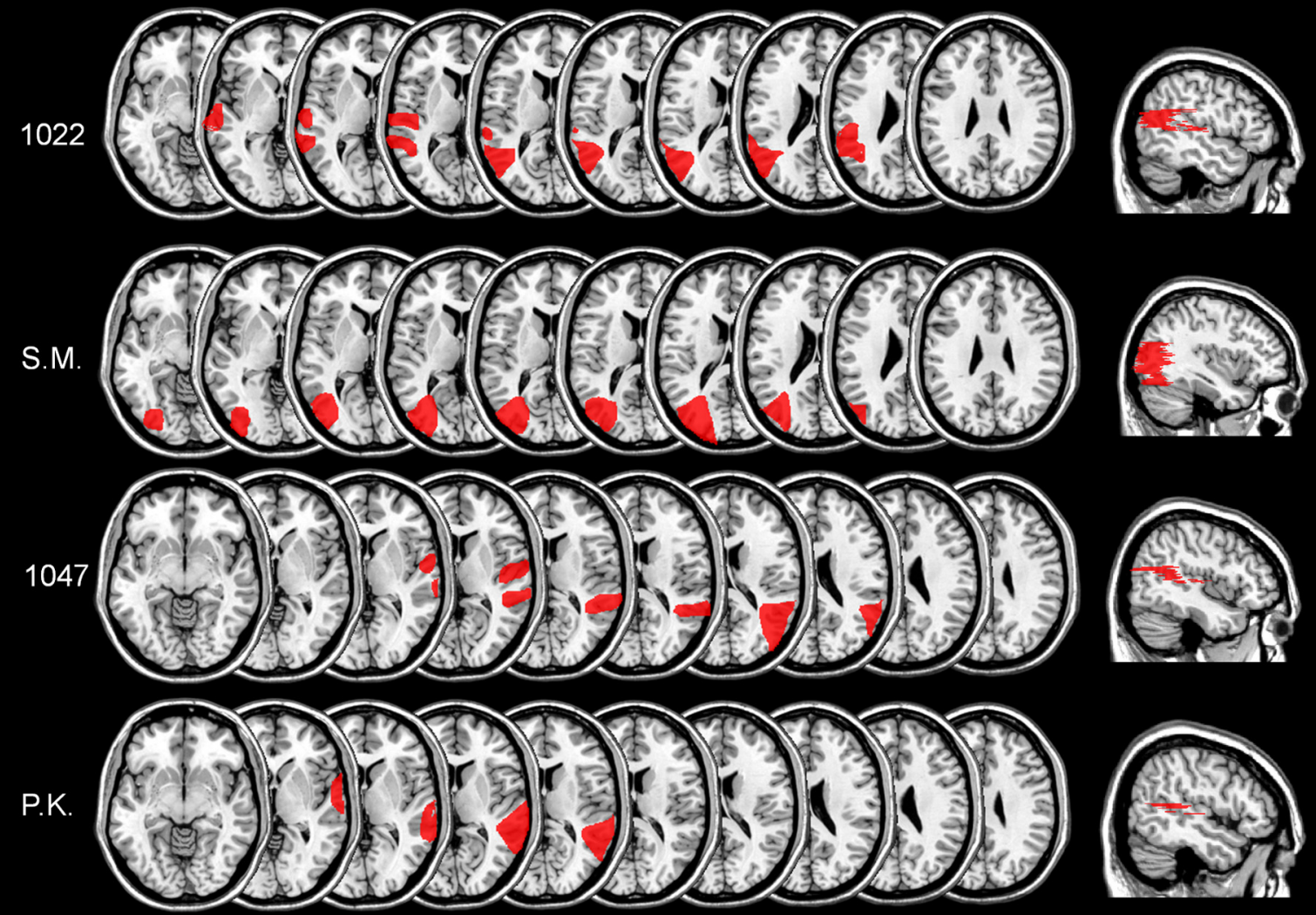

Figure 2. Individual patients' lesions. DPC patients had lesions in the dorsal parietal cortex; VPC patients had lesions in the ventral parietal cortex. L, Left; $R$, right.

a within-subject factor yielded a significant effect of condition $\left[F_{(3,39)}=6.61 ; p<0.001\right]$. Post hoc comparisons, performed with the Scheffé test, indicated that hits were more frequent in the Intact compared with the Recombined condition $(p<0.05)$, the
NewOld condition $(p<0.01)$, and, marginally, the NoOld condition $(p=0.06)$. No significant difference emerged among the Recombined, NewOld, and NoOld conditions $(p>0.16$ in all cases). The same ANOVA conducted on RTs yielded a significant 

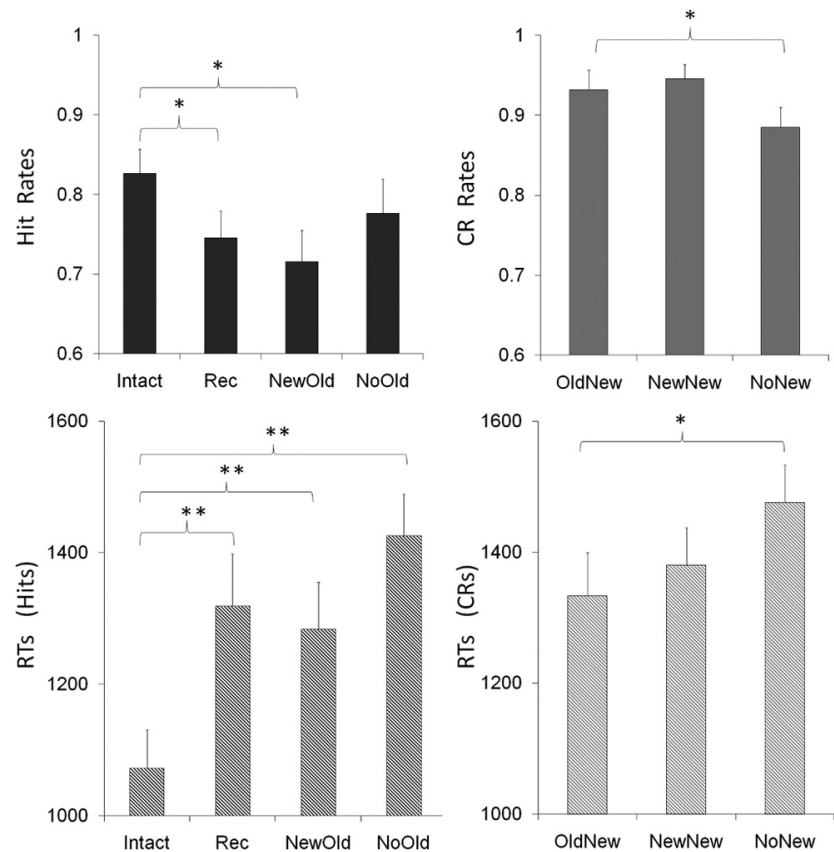

Figure 3. Mean frequency and response times (RTs) for hits (left panel) and correct rejections (right panel) by condition. Error bars indicate SEM. CR, Correct rejection; Rec, Recombined. ${ }^{*} p<0.05 ;{ }^{* *} p<0.005$.

effect of condition $\left[F_{(3,39)}=24.11 ; p<0.0005\right]$ : hits were faster in the Intact compared with all the other conditions $(p<0.005$ in all cases), but there were no significant differences among the Recombined, NewOld, and NoOld conditions $(p>0.08$ in all cases).

The ANOVA on the proportion of correct rejections with condition (OldNew, NewNew, NoNew) as a within-subject factor yielded a significant effect of condition $\left[F_{(2,26)}=3.84 ; p<0.05\right]$. Post hoc comparisons indicated that correct rejections were more frequent in the OldNew compared with the NoNew condition $(p<0.05)$, but not significantly different between the OldNew and the NewNew conditions $(p=0.56)$. The same ANOVA conducted on RTs yielded a significant effect of condition $\left[F_{(2,26)}=3.37 ; p<0.05\right]$ : correct rejections were faster in the OldNew compared with the NoNew condition $(p<0.05)$, but not different between the OldNew and the NewNew conditions $(p=0.41)$.

Consistent with our predictions, subjects were faster and more accurate at recognizing old words in the Intact compared with the NoOld, NewOld, and Recombined condition. Subjects were also faster and more accurate at rejecting new words in the OldNew compared with the NoNew condition, suggesting an effect of memory cueing on correct rejections. As much as a match between mnemonic expectations and a memory probe helped classify that probe as "old" (Intact condition), a mismatch between mnemonic expectations and a probe might have favored a "new" response (OldNew condition). Such effect, however, was less consistent than that on hits, as we failed to find a difference between the OldNew and the NewNew condition. Overall, the results indicate that participants developed mnemonic expectations during the cue period, which facilitated subsequent recognition decisions.

Overall retrieval success

Table 1 shows brain regions that exhibited more activity for hits (collapsed across the Intact, Recombined, NoOld, NewOld con-
Table 1. Regions showing retrieval success effects

\begin{tabular}{lrrrrrr}
\hline & & \multicolumn{2}{c}{ Peak coordinates $^{a}$} & No. of \\
\cline { 3 - 4 } Region & BA & $x$ & $Y$ & $Z$ & voxels & $t$ value \\
\hline Parietal & & & & & & \\
$\quad$ Precuneus & 19 & -36 & -66 & 42 & 214 & 4.5 \\
$\quad$ Postcentral gyrus & 3 & 20 & -38 & 66 & 44 & 4.4 \\
$\quad$ Supramarginal gyrus & 40 & -60 & -32 & 30 & 19 & 4.3 \\
Frontal & & & & & & \\
$\quad$ Orbitofrontal cortex & 11 & -36 & 50 & -10 & 823 & 4.3 \\
$\quad$ Dorsolateral prefrontal cortex & 9 & -38 & 10 & 36 & 21 & 4.3 \\
$\quad$ Premotor cortex & 6 & -26 & 10 & 56 & 81 & 6.2 \\
$\quad$ Cingulate gyrus & 24 & 0 & -4 & 26 & 39 & 4.6 \\
Limbic & & & & & & \\
$\quad$ Parahippocampal gyrus & 27 & -10 & -36 & 2 & 32 & 4.2 \\
$\quad$ Posterior cingulate & 30 & 20 & -62 & 8 & 38 & 4.5 \\
$\quad$ Sublobar & & & & & & \\
$\quad$ Insula & 13 & 46 & -30 & 22 & 31 & 4.2 \\
\hline Talairach and Tournoux (1988). & & & & & &
\end{tabular}

Table 2. Regions showing effects related to top-down AtoM

\begin{tabular}{|c|c|c|c|c|c|c|}
\hline \multirow[b]{2}{*}{ Region } & \multirow[b]{2}{*}{$\mathrm{BA}$} & \multicolumn{3}{|c|}{ Peak coordinates $^{a}$} & \multirow{2}{*}{$\begin{array}{l}\text { No. of } \\
\text { voxels }\end{array}$} & \multirow[b]{2}{*}{$t$ value $^{b}$} \\
\hline & & $x$ & $y$ & $z$ & & \\
\hline \multicolumn{7}{|l|}{ Parietal } \\
\hline Intraparietal sulcus & 7 & -34 & -56 & 42 & 14 & 3.12 \\
\hline \multicolumn{7}{|l|}{ Frontal } \\
\hline Dorsolateral prefrontal cortex & 46 & -46 & 28 & 16 & 144 & 3.81 \\
\hline Premotor cortex & 6 & -32 & 2 & 38 & 79 & 4.38 \\
\hline Ventrolateral prefrontal cortex & 47 & -32 & 24 & -4 & 28 & 3.35 \\
\hline
\end{tabular}

Talairach and Tournoux (1988).

${ }^{b}$ The $t$ value reported for peak regions in a conjunction analysis is the minimum $t$ value among the contrasts used as inclusive masks.

ditions) than for correct rejections (collapsed across the OldNew, NoNew, NewNew conditions). In line with previous findings, retrieval success was related to activation in left inferior parietal cortex, left precuneus, and posterior cingulate cortex, as well as in the left middle frontal gyrus, including both dorsolateral and orbitofrontal regions, and the left parahippocampal gyrus (Konishi et al., 2000; McDermott et al., 2000; Wheeler and Buckner, 2003). Consistent with previous reviews (Ciaramelli et al., 2008), activity in PPC related to retrieval success involved both a left DPC cluster centered on the precuneus and extending to the intraparietal region and a left VPC cluster centered in the supramarginal gyrus.

\section{Top-down AtoM}

The conjunction analysis to identify regions simultaneously active in the contrasts (Intact - NoOld) AND (Recombined NoOld) AND (OldNew - NoNew), while excluding those active in the contrasts (NewOld - NoOld) AND (NewNew - NoNew), revealed brain regions associated with top-down AtoM. These are reported in Table 2 .

In line with our predictions, these regions included a left DPC region centered in the anterior intraparietal sulcus (IPS; $x=-34$, $y=-56, z=42$ ) (Fig. 4). The IPS site related to top-down AtoM in the present study overlapped strikingly with the center of mass of activations related to top-down AtoM $(x=-36, y=-57, z=$ 42) (Ciaramelli et al., 2008). In addition to the DPC, activity related to top-down AtoM was found in dorsolateral prefrontal cortex (DLPFC; BA 46/9), ventrolateral prefrontal cortex (VLPFC; BA 47), and premotor cortex (BA 6).

Activity in regions implicated in top-down AtoM was expected to predict the degree to which individuals were facilitated in target detection in the Intact condition, i.e., when memory was (validly) cued, compared with the NoOld condition, i.e., when 


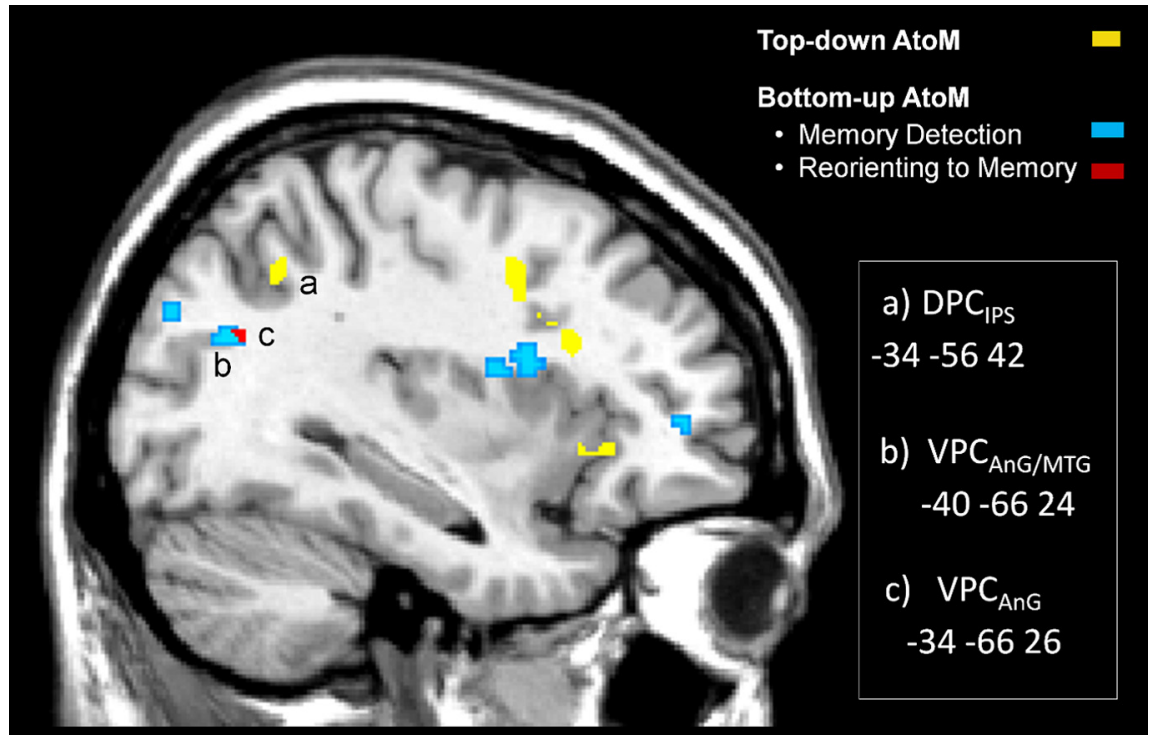

Figure 4. Brain regions mediating top-down AtoM and bottom-up AtoM. Top-down AtoM regions included a left dorsal parietal region in the intraparietal sulcus (DPC IPS) ( $\boldsymbol{a}$ ). Bottom-up AtoM regions included a left ventral parietal region at the border between the angular gyrus and the middle temporal gyrus, mediating detection of memory targets $\left(\mathrm{VPC}_{\mathrm{AnG} / \mathrm{MTG}}\right)(\boldsymbol{b})$, and a region in the left angular gyrus specialized for detection of unexpected memory targets $\left(\mathrm{VPC}_{\mathrm{AnG}}\right)$ (c).

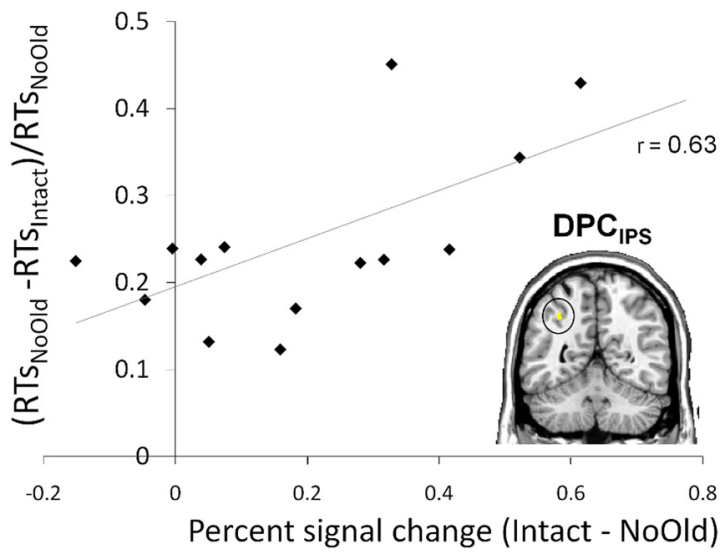

Figure 5. Scatterplot of the correlation between advantage in RTs in the Intact versus NoOld condition and difference in percentage signal change between the two conditions for dorsal parietal cortex (DPC $\mathrm{IPS}_{\mathrm{S}}$ ).

Table 3. Regions showing effects related to bottom-up AtoM

\begin{tabular}{|c|c|c|c|c|c|c|}
\hline \multirow{2}{*}{ Region } & \multirow[b]{2}{*}{ BA } & \multicolumn{3}{|c|}{ Peak coordinates $^{a}$} & \multirow{2}{*}{$\begin{array}{l}\text { No. of } \\
\text { voxels }\end{array}$} & \multirow[b]{2}{*}{$t$ valu } \\
\hline & & $x$ & $y$ & $z$ & & \\
\hline \multicolumn{7}{|l|}{ Memory detection } \\
\hline \multicolumn{7}{|l|}{ Parietal } \\
\hline Angulargyrus/middle temporal gyrus & 39 & -40 & -66 & 24 & 33 & 4.2 \\
\hline Postcentral gyrus & 3 & 20 & -32 & 60 & 40 & 4.3 \\
\hline \multicolumn{7}{|l|}{ Frontal } \\
\hline Dorsolateral prefrontal cortex & 9 & -46 & 10 & 26 & 63 & 4.2 \\
\hline Lateral anterior prefrontal cortex & $10 / 46$ & -42 & 44 & 6 & 32 & 4.4 \\
\hline Premotor cortex & 6 & -26 & 10 & 56 & 76 & 4.2 \\
\hline \multicolumn{7}{|l|}{ Temporal } \\
\hline Transverse temporal gyrus & 42 & 60 & -12 & 12 & 31 & 4.8 \\
\hline \multicolumn{7}{|l|}{ Occipital } \\
\hline Superior occipital gyrus & 19 & -32 & -80 & 32 & 25 & 4.3 \\
\hline \multicolumn{7}{|l|}{ Reorienting to memory } \\
\hline \multicolumn{7}{|l|}{ Parietal } \\
\hline Angular gyrus & 39 & -34 & -66 & 26 & 7 & $2.5^{b}$ \\
\hline
\end{tabular}

Talairach and Tournoux (1988).

${ }^{b}$ The $t$ value reported for peak regions in a conjunction analysis is the minimum $t$ value among the contrasts used as inclusive masks. memory was not cued. To test this hypothesis, we ran a Pearson correlation analysis between the difference in percentage signal change between the Intact and the NoOld conditions and the magnitude of the cueing effect on accuracy and on RTs. An index of relative increase in recognition accuracy in the Intact compared with the NoOld condition was computed as (Accuracy $_{\text {Intact }}-$ Ac-

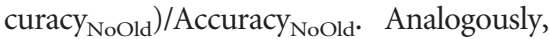
an index of relative decrease in RTs in the Intact compared with the NoOld condition was computed as $\left(\mathrm{RT}_{\text {NoOld }}-\mathrm{RT}_{\text {Intact }}\right) / \mathrm{RT}$ NoOld. We found that the cueing effect on RTs correlated significantly with activity in DPC ( $\mathrm{r}=0.63, p<0.05)$ (Fig. 5) and $\operatorname{VLPFC}(\mathrm{r}=0.54, p<0.05)$ : The more subjects activated these regions upon presentation of a relevant memory cue, the faster they were in detecting the upcoming memory target.

\section{Bottom-up AtoM}

Memory detection. Table 3 shows brain regions that responded more strongly to memory targets than to new items when no cue was provided (NoOld - NoNew). In line with the predictions, Memory Detection was associated with activity in a left VPC region, at the border between the angular gyrus (AnG) and the middle temporal gyrus (MTG) $\left(\mathrm{VPC}_{\mathrm{AnG} / \mathrm{MTG}} ; x=-40, y=\right.$ $-66, z=24$ ) (Fig. 4). In addition to VPC, the contrast revealed activity in premotor cortex (BA 6), DLPFC (BA 9), and lateral anterior prefrontal cortex (BA 10/46).

Activity in regions implicated in bottom-up Memory Detection was expected to predict subjects' speed and ability to recognize targets for memory. To test this hypothesis, we ran a Pearson correlation analysis between percentage signal change and hit rates in the NoOld condition. Moreover, by using a simple regression analysis, we looked for regions whose activity correlated with RTs in the NoOld condition. We found that hit rates correlated significantly with brain activity in $\mathrm{VPC}_{\mathrm{AnG} / \mathrm{MTG}}(r=0.56, p<$ 0.05 ) (Fig. 6). Moreover, activity in a region overlapping with $\mathrm{VPC}_{\text {AnG/MTG }}$ (peak: $x=-36, y=-66, z=24$ ) correlated negatively with RTs in the NoOld condition ( $p<0.05$, uncorrected). Thus, when no cue was provided, the more subjects activated the left VPC upon presentation of memory targets, the more efficient and faster was target detection, consistent with the hypothesis that VPC mediates the capture of bottom-up attention by memory contents. In contrast, activity in a cluster overlapping with our DLPFC region (peak: $x=-46, y=8, z=26$ ) correlated positively with RTs in the NoOld condition ( $p<0.05$, uncorrected), suggesting that this region was not related to bottom-up AtoM but rather to detection of memory targets that were difficult to identify.

Reorienting to memory. The conjunction analysis to identify regions active in the contrasts (Recombined - Intact) AND (NoOld - NoNew), while excluding those active in the contrast (Recombined - OldNew), unveiled a left VPC region in the angular gyrus $\left(\mathrm{VPC}_{\mathrm{AnG}} ; x=-34, y=-66, z=26\right)$ associated with detection of targets that were invalidly compared with validly cued (Table 3; Fig. 4). An ANOVA $\left[F_{(3,39)}=2.96 ; p<0.05\right]$ confirmed that activity in $\mathrm{VPC}_{\mathrm{AnG}}$ was higher in the Recombined (0.09) compared with the Intact (0.02), NoOld (0.04), and NewOld $(0.04)$ conditions $\left(p<0.05\right.$ in all cases). That is, activity in $\mathrm{VPC}_{\mathrm{AnG}}$ 


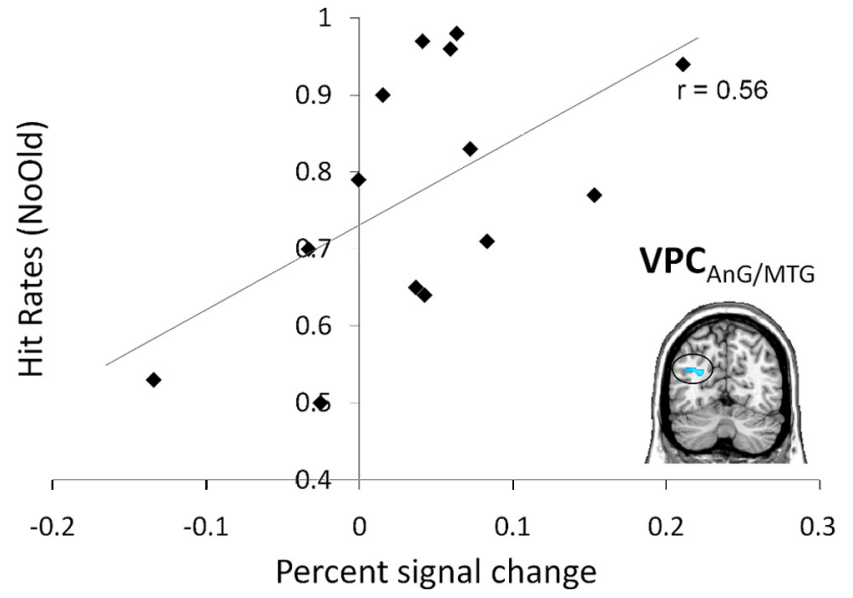

Figure 6. Scatterplot of the correlation between hit rates and percentage signal change in the NoOld condition for ventral parietal cortex (VPC $\left.\mathrm{AnG}_{\mathrm{AMTG}}\right)$.

increased when mnemonic expectations were violated (Recombined condition) relative to when mnemonic expectations were met (Intact condition) or not formed at all (NoOld and NewOld conditions), consistent with the hypothesis that this region may mediate reorienting of attention to unexpected memory targets. In contrast, activity in $\mathrm{VPC}_{\mathrm{AnG}}$ did not discriminate between the Intact and the NewOld conditions $(p=0.57)$ despite the significantly higher hit rates in the former compared with the latter, further suggesting that this region is not sensitive to the amount of retrieved information.

Activity in regions associated with detection of unexpected targets was postulated to predict subjects' ability to recognize memory targets that were invalidly cued. We found no significant correlation, however, between percentage signal change in $\mathrm{VPC}_{\mathrm{AnG}}$ and hit rates $(r=-0.19)$ or RTs $(r=-0.18)$ in the Recombined condition ( $p>0.49$ in both cases). Let us consider possible reasons for the absence of the predicted relation. One possibility is that activity associated with reorienting to unexpected memory targets reflected a sum of signals indexing breaches of expectations and reflexive shifts of attention to unexpected targets (Shulman et al., 2009). While activity related to each signal in isolation may be related to behavior, averaging brain activity across the whole trial could have weakened our ability to detect relations between brain and behavior. Another possibility is that VPC mediates a transient switch signal promoting reorienting of attention to unexpected targets, but the actual shift of attention is performed by a dorsal frontoparietal network (Corbetta et al., 2008, Shulman et al., 2009). In this case, performance in the Recombined condition may be related to the ability of the VPC signal to recruit the dorsal frontoparietal network or to the sustained activity of this network, rather than to the magnitude of the VPC signal per se. Interestingly, activity in DPC correlated positively, although not significantly, with hit rates in the Recombined condition $(r=0.45 ; p=0.10)$. Studies investigating the functional connectivity of VPC during detection of unexpected targets will help to elucidate the whole network involved in shifting attention to unexpected memories and its relation with behavior. In any event, if VPC mediates signals necessary to detect unexpected targets, patients with lesions in VPC should be disproportionately impaired at detecting memory targets in the Recombined condition compared with their basic recognition performance. We test this prediction in the neuropsychological study.
Summary of functional dissociations between top-down and bottom-up AtoM regions

To confirm further the functional dissociations among DPC and VPC regions, we compared activity in these regions across four relevant conditions (Fig. 7). We ran an ANOVA on percentage signal change with ROI (DPC, $\left.\mathrm{VPC}_{\mathrm{AnG} / \mathrm{MTG}}, \mathrm{VPC}_{\mathrm{AnG}}\right)$ and condition (Intact, Recombined, NoOld, NoNew) as factors. The results revealed a significant effect of ROI $\left[F_{(2,26)}=3.80, p<0.05\right]$, condition $\left[F_{(3,39)}=16.96, p<0.000005\right]$, and ROI $\times$ condition interaction $\left[F_{(6,78)}=4.97, p<0.0005\right]$. Post hoc comparisons showed that activity in DPC distinguished cued versus noncued memory targets (Intact vs NoOld condition; $t_{(13)}=3.32, p<$ 0.01 ) but not memory targets versus nontargets (NoOld vs NoNew condition; $p=0.23$ ) or unexpected versus expected targets (Recombined vs Intact condition; $p=0.82$ ). By contrast, $\mathrm{VPC}_{\mathrm{AnG} / \mathrm{MTG}}$ distinguished memory targets versus nontargets $\left(t_{(13)}=5.73, p<0.001\right)$ but not cued versus noncued targets $(p=0.94)$ or unexpected versus expected targets $(p=0.16)$. Finally, $\mathrm{VPC}_{\mathrm{AnG}}$ distinguished memory targets versus nontargets $\left(t_{(13)}=4.53 ; p<0.001\right)$ and responded more strongly to unexpected versus expected targets $\left(t_{(13)}=2.94 ; p<0.05\right)$ but did not distinguish cued from noncued targets $(p=0.57)$.

Additionally, we followed up on correlation analyses relating brain activity with behavioral performance and investigated the selectivity of DPC and VPC regions in predicting behavioral effects related to top-down and bottom-up AtoM, respectively (supplemental Table 3s, available at www.jneurosci.org as supplemental material). Whereas activity in DPC correlated with the effect of memory cueing on RTs (Fig. 5), activity in VPC regions was not related to the effect of memory cueing on either RTs (for $\mathrm{VPC}_{\mathrm{AnG} / \mathrm{MTG}}: r=0.13, p=0.65$; for $\mathrm{VPC}_{\mathrm{AnG}}: r=-0.20, p=$ 0.50 ) or recognition accuracy (for $\mathrm{VPC}_{\mathrm{AnG} / \mathrm{MTG}}: \mathrm{r}=0.07, p=$ 0.79; for $\left.\mathrm{VPC}_{\mathrm{AnG}}: r=-0.09, p=0.74\right)$. On the other hand, whereas activity in $\mathrm{VPC}_{\mathrm{AnG} / \mathrm{MTG}}$ correlated with hit rates in the NoOld condition (Fig. 6), activity in DPC did not correlate with hit rates $(r=0.09 ; p=0.75)$ or RTs $(r=-0.13 ; p=0.64)$ in the NoOld condition. Neither VPC nor DPC activity correlated significantly with recognition performance in the Recombined condition, although DPC activity tended to increase with increasing hit rates $(r=0.45 ; p=0.10)$.

Together, these analyses confirm that activity in VPC was mainly associated with bottom-up AtoM effects, whereas activity in DPC was mainly associated with top-down AtoM effects.

\section{Spatial overlap between retrieval success and AtoM regions}

We investigated formally whether DPC and VPC regions related to top-down AtoM and bottom-up AtoM are subsets of those regions exhibiting retrieval success effects or rather belong to partially distinct neural systems. To assess the spatial overlap between retrieval success and top-down AtoM regions, we ran a conjunction analysis between the Overall retrieval success map and the top-down AtoM map as obtained in the previous sections, i.e., with a level of significance of $p<0.001$ for both the Overall retrieval success map and the top-down AtoM map. There was no overlap between the two maps. A region of overlap was detected when the level of significance of both maps was lowered to $p<0.005$, which was located between the IPS and the precuneus (peak: $x=-30, y=-64, z=34$ ). Analogously, we investigated the spatial overlap between retrieval success and bottom-up AtoM regions. First, we ran a conjunction analysis between the Overall retrieval success map and the Memory Detection map as obtained in the previous sections, i.e., with a level of significance of $p<0.001$ for both maps. There was no overlap 
$\mathrm{DPC}_{\mathrm{IPS}}$

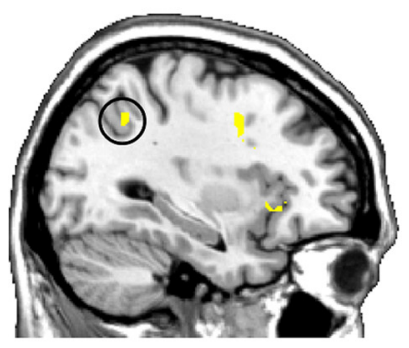

\section{VPC $_{\text {AnG/MTG }}$}

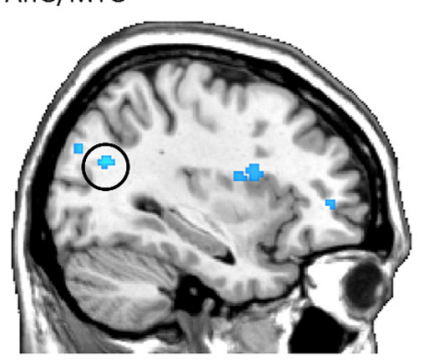

$\mathrm{VPC}_{\text {AnG }}$

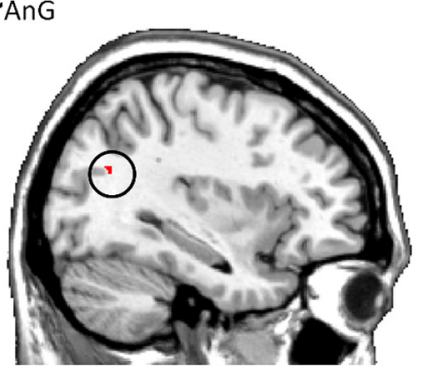

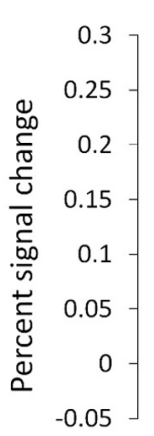

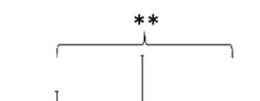

- Intact
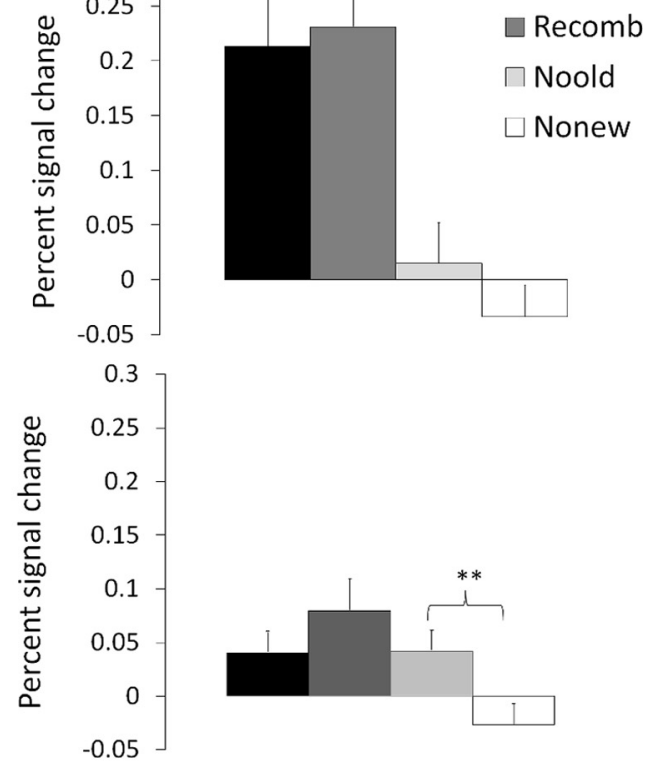

0.3

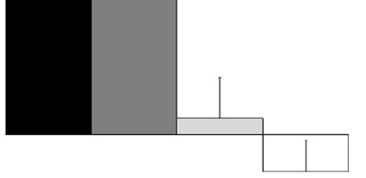

$\checkmark$ Nonew
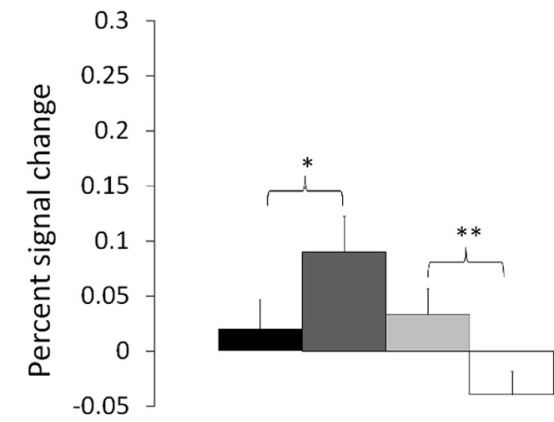

To summarize, the fMRI study revealed that DPC was activated when participants searched for/anticipated a target based on a relevant cue, and its activity was correlated with the behavioral advantage from memory cueing. In contrast, activation in VPC regions predicted efficacy and speed of target detection when no cue was provided $\left(\mathrm{VPC}_{\mathrm{AnG} / \mathrm{MTG}}\right)$ and was strongest for unexpected memory targets $\left(\mathrm{VPC}_{\mathrm{AnG}}\right)$. These results support the hypothesis that DPC and VPC mediate topdown and bottom-up AtoM, respectively.

\section{Neuropsychological study}

To confirm the conclusions we drew from our neuroimaging data by providing converging evidence, we tested patients with lesions centered on the VPC or the DPC using the same paradigm. Figure 8 shows the overlap between brain lesions in DPC and VPC patients and regions implicated in top-down and bottom-up AtoM detected in the fMRI study, respectively.

If DPC is crucial to support top-down AtoM, then DPC patients should show a reduced benefit from valid memory cueing compared with normal controls. On the other hand, if VPC is crucial to support bottom-up AtoM, then VPC patients should be impaired compared with the controls at detecting memory targets when no cue is provided (NoOld condition). Moreover, if VPC mediates reorienting of attention to unexpected targets, then VPC patients should be disproportionately impaired at detecting memory

Figure 7. Percentage signal change by condition in dorsal (DPC IPS $)$ and ventral parietal regions $\left(\mathrm{VPC}_{\mathrm{AnG} / \mathrm{MTG}}, \mathrm{VPC}_{\mathrm{AnG}}\right)$. Recomb, Recombined. Bars represent SEs of the mean. ${ }^{*} p<0.05 ;{ }^{* *} p<0.01$.

between the two maps. A region of overlap was detected when the level of significance of both maps was lowered to $p<0.005$, which was located in BA 39 (peak: $x=-42, y=-66, z=26$ ). Next, we ran a conjunction analysis between the Overall retrieval success map and the Reorienting to Memory map as obtained in the previous sections, i.e., with a level of significance of $p<0.001$ for both the Overall retrieval success map and the Reorienting to Memory map. There was no overlap between the two maps. A region of overlap was detected when the level of significance of both maps was lowered to $p<0.005$, which was located in BA 39 (peak: $x=-36, y=-64, z=28$ ).

These findings suggest that, although there is some overlap between regions associated with retrieval success and AtoM regions, the latter regions are not specialized for retrieval success but rather for providing attentional support to retrieval operations. Their involvement in retrieval success, therefore, is likely not unconditional but critically may depend on the degree to which the memory task poses demands on top-down and bottom-up AtoM. Indeed, retrieval success effects in PPC have been found to move toward DPC as the difficulty of the recognition task increases (Cabeza et al., 2008). If AtoM regions are partially segregated from retrieval success regions, it should be possible to find patients with lesions overlapping with AtoM regions that show deficits in top-down and bottom-up AtoM in the absence of a generalized impairment of recognition memory. We test this prediction in the neuropsychological study. targets in the Recombined condition compared with their basic recognition performance.

Due to the small sample sizes, performance differences between patients and controls were assessed by means of nonparametric statistics (Mann-Whitney $U$ test). We also report on relevant differences in the behavior of left- and right-lesioned patients. Because there was only one patient with right DPC lesions, behavioral measures for this patient were analyzed using individual modified $t$ tests based on the method of Crawford and Garthwaite (2002) for comparing single cases to small control samples.

\section{Recognition accuracy}

Across conditions, recognition accuracy was calculated as hit rates minus false alarm rates. The frequency of false alarms in the OldNew condition served as false alarm rate for both the Intact and the Recombined condition (see supplemental Table 4s, available at www.jneurosci.org as supplemental material, for mean hit rates, correct rejection rates, accuracy, and median RTs by group and condition).

Recognition accuracy was not significantly different between VPC patients and controls across conditions $(p>0.12$ in all cases). Across conditions, however, RTs to both hits (MannWhitney $U<4$; $p<0.05$ in all cases) and correct rejections (Mann-Whitney $U<5$; $p<0.05$ in all cases) were longer in VPC patients than in controls. There was no significant difference be- 
tween patients with lesions to left versus right VPC, either in memory accuracy or RTs ( $p>0.12$ in all cases). A different pattern of results emerged for DPC patients. Recognition accuracy was significantly impaired, compared with the controls, in the Intact condition (MannWhitney $U=4 ; p<0.05$ ) but not in the other conditions ( $p>0.18$ in all cases). Similarly, DPC patients were significantly slower at detecting targets than controls in the Intact condition (Mann-Whitney $U=4 ; p<0.05)$ but not in the other conditions $(p>0.17$ in all cases). The same pattern of results emerged when statistical analyses were run excluding patient 1051. There was no significant difference between patients with lesions to left versus right DPC, either in memory accuracy or RTs ( $p>0.17$ in all cases).

These findings indicate that VPC patients were not impaired at distinguishing studied from new items, although they needed more time than the controls to make memory decisions. On the other hand, DPC patients were impaired only in the Intact condition, that is, when normal controls benefited from memory cueing, suggesting that they might have a selective deficit in top-down AtoM.

\section{Top-down AtoM}

We then investigated more precisely the extent to which patients and controls ben-

efited from memory cueing by examining performance in the Intact (validly cued) condition relative to basic recognition performance in the NoOld (noncued) condition. For each participant, an index of relative change in recognition accuracy in the Intact compared with the NoOld condition was computed as (Accuracy In $^{-}$ tact - Accuracy $\left._{\text {NoOld }}\right) /$ Accuracy $_{\text {NoOld }}$. Analogously, an index of relative change in RTs in the Intact compared with the NoOld condition was computed as $\left(\mathrm{RT}_{\text {Intact }}-\mathrm{RT}_{\text {NoOld }}\right) / \mathrm{RT}_{\text {NoOld}}$.

We found that in the Intact compared with the NoOld condition accuracy increased less (Mann-Whitney $U=2 ; p<0.05$ ), and RTs decreased less (Mann-Whitney $U=1 ; p<0.05$ ) in DPC patients than in controls (Fig. 9). The reduction in the advantage on recognition accuracy from valid memory cueing compared with the controls was statistically significant for left DPC patients (Mann-Whitney $U=2 ; p<0.05$ ) but only marginally significant for the right DPC patient $(t=-1.39, p=0.10)$. The reduction in the advantage on RTs from valid memory cueing compared with the controls was statistically significant for both left DPC patients (Mann-Whitney $U=0 ; p<0.05$ ) and the right DPC patient $(t=$ $-2.45, p<0.05)$. Removing patient 1051 from the analyses did not alter the results. In contrast, VPC patients, both those with left and those with right lesions, benefited from memory cueing to the same extent as did normal controls, both in terms of accuracy and RTs ( $p>0.43$ in all cases). Analogous results were obtained if the index of relative change in performance in the Intact (validly cued) condition was computed relative to the NewOld condition (i.e., the other noncued condition) as opposed to the NoOld condition (supplemental Table $4 \mathrm{~s}$ and supplemental Results, available at www.jneurosci.org as supplemental material).

These findings indicate that DPC patients did not benefit from valid memory cueing as much as the controls, consistent with the hypothesis that DPC supports top-down AtoM. The neuropsychological evidence suggests that both right and left DPC may be crucial for top-down AtoM, although activation in right DPC was not detected in the fMRI study.

\section{Bottom-up AtoM}

We next investigated whether patients and controls were equally able to detect memory contents after invalid memory cueing by examining performance in the Recombined (invalidly cued) condition relative to basic recognition performance in the NoOld (noncued) condition. For each participant, an index of relative change in hit rates in the Recombined compared with the NoOld

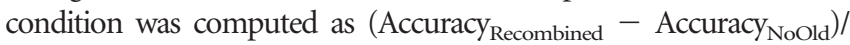
Accuracy $_{\text {NoOld}}$. Analogously, an index of relative change in RTs in the Recombined compared with the NoOld condition was computed as $\left(\mathrm{RTs}_{\text {Recombined }}-\mathrm{RTs}_{\text {NoOld }}\right) / \mathrm{RTs}_{\text {NoOld }}$

The index of relative change in hit rates in the Recombined compared with the NoOld condition was comparable across groups ( $p>0.22$ in all cases). However, RTs increased more in the Recombined compared with the NoOld condition in VPC patients than in controls (Mann-Whitney $U=4 ; p<0.05$ ) (Fig. 10). VPC patients' deficit in the Recombined condition was driven by patients with left VPC lesions who suffered from invalid memory cueing significantly more than the controls (Mann-Whitney $U=0 ; p<0.05$ ), whereas right VPC patients did not $(p>0.29)$. In contrast, no significant difference emerged 


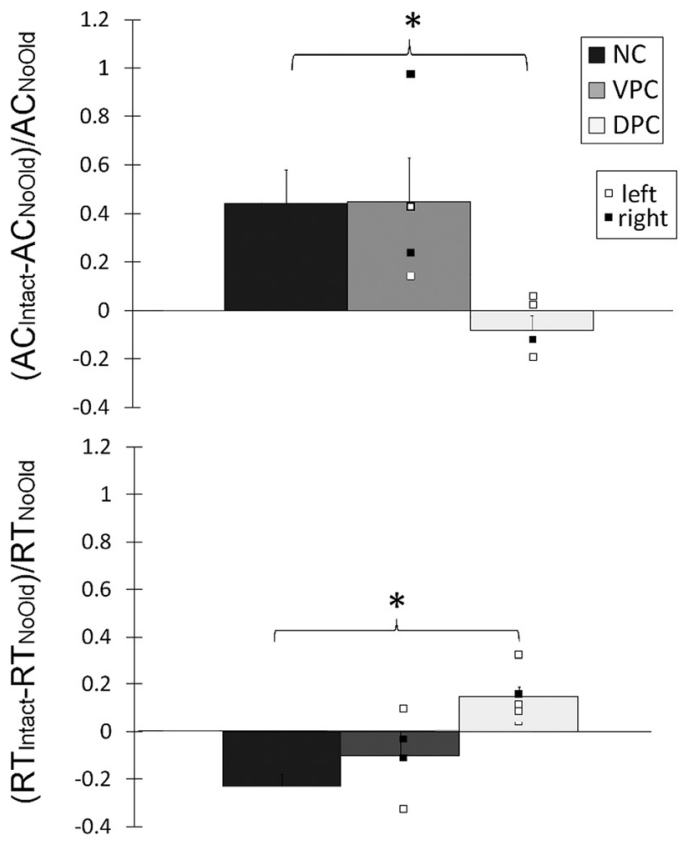

Figure 9. Lack of behavioral advantage in the Intact (validly cued) versus NoOld (noncued) condition on recognition accuracy (AC; top) and RTs (bottom) in patients with DPC lesions compared with patients with VPC lesions and healthy controls (NC). White squares denote performance of individual patients with left lesions, and black squares that of individual patients with right lesions. Error bars indicate SEM. ${ }^{*} p<0.05$.

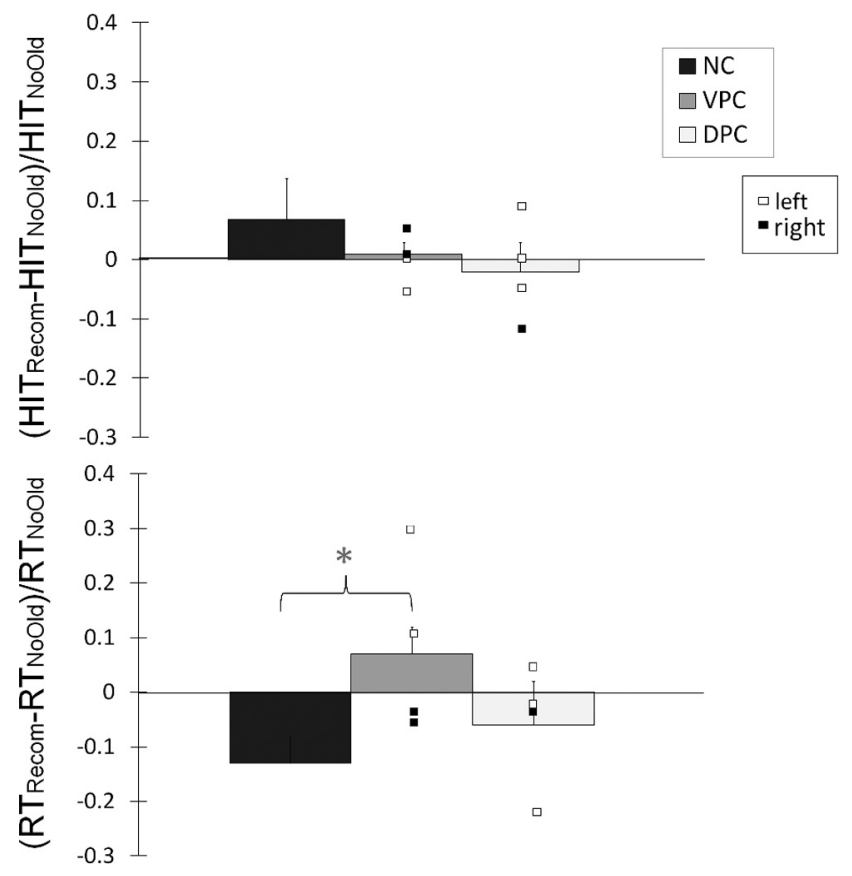

Figure 10. Behavioral disadvantage in the Recombined (invalidly cued) versus NoOld (noncued) condition on hit rates (HIT; top) and RTs (bottom) in patients with VPC lesions, compared with patients with DPC lesions, and healthy controls (NC). White squares denote performance of individual patients with left lesions, and black squares denote performance of individual patients with right lesions. Error bars indicate SEM. ${ }^{*} p<0.05$.

between DPC patients, both those with left and right lesions and controls ( $p>0.25$ in both cases). Analogous results were obtained if the index of relative change in performance in the Recombined (invalidly cued) condition was computed relative to the NewOld (i.e., the other noncued condition) or the Intact (i.e., validly cued) condition, as opposed to the NoOld condition (supplemental Table $4 \mathrm{~s}$ and supplemental Results, available at www. jneurosci.org as supplemental material). However, when the relative change in performance was computed relative to the Intact condition, DPC patients exhibited extremely low reorienting costs in the Recombined condition compared with controls. In these patients, DPC lesions arguably prevented the development of mnemonic expectations and, in turn, the benefit from valid memory cueing in the Intact condition, thus accounting for the absence of reorienting costs after invalid cueing (Recombined condition).

In line with the fMRI results, these findings indicate that patients with lesions in left VPC had problems detecting unexpected memories, consistent with the hypothesis that left VPC supports bottom-up AtoM.

\section{Discussion}

Using a novel recognition memory task and combining functional neuroimaging and lesion approaches, we tested the hypothesis that DPC and VPC are related to top-down and bottom-up AtoM, respectively (Cabeza et al., 2008; Ciaramelli et al., 2008). Our results provide converging evidence supporting the AtoM hypothesis, showing that DPC was associated with search for/orienting to memory contents, whereas VPC was associated with detection of memory contents, especially when they were unexpected.

\section{Top-down AtoM}

Two findings support a role for the DPC in top-down AtoM. First, a cluster in left DPC, centered on the anterior IPS, was more active when participants searched for/anticipated upcoming memory targets upon presentation of memory cues than when no cue was provided. Importantly, the level of activation of DPC on cued trials predicted participants' advantage in detecting cued versus noncued memory targets. In contrast, DPC activity did not significantly distinguish studied from new words in noncued trials. These functional properties support the hypothesis that, despite not having apparent mnemonic properties, DPC allocates attentional resources to processes that assist memory retrieval, such as memory search (Wagner et al., 2005; Cabeza et al., 2008). Consistently, effortful memory decisions, which require sustained retrieval attempts, activate DPC more than easy memory decisions (Cabeza et al., 2008), and DPC activity decreases over repeated retrieval attempts (Kuhl et al., 2007). Moreover, DPC regions support orienting of attention to memories with particular properties (Wheeler et al., 2006).

The neuroimaging findings were confirmed by neuropsychological evidence. Despite a general preservation of recognition memory, patients with lesions in DPC did not benefit from memory cueing as did patients with lesions limited to the VPC and normal controls. The selective deficit in memory cueing in DPC patients, in the face of generally preserved recognition memory, is consistent with the hypothesis that patients could not initiate an efficient memory search upon presentation of relevant cues because of impaired top-down AtoM. Of course, reduced memory cueing in DPC patients might also be explained with a pure recollection deficit. If patients successfully initiated a memory search but failed at recalling the paired associate of relevant cues, memory cueing would not occur. If DPC simply mediated recollection, however, one might expect activity in this region to be larger for studied than for new words. Our finding from the fMRI study that DPC activity was not significantly different in the NoOld compared with the NoNew condition is more consistent 
with a top-down AtoM account of DPC. In addition, patients with lesions in DPC can succeed in memory tasks requiring recollection (Davidson et al., 2008, Simons et al., 2008), consistent with the idea that DPC does not mediate recollection per se.

In addition to DPC, left DLPFC, VLPFC, and lateral premotor cortex also showed activity related to top-down AtoM. DLPFC has long been known to support top-down attentional control (Miller and Cohen, 2001), and lateral premotor cortex has been implicated in attentional cueing (Cristescu et al., 2006). Left DLPFC and lateral premotor cortex might then act in concert with DPC to support top-down AtoM. Accordingly, DLPFC supports effortful memory decisions (Henson et al., 2000; Fleck et al., 2006; Kim and Cabeza, 2007), as does DPC. Moreover, activity in lateral premotor cortex was found to modulate depending on the search instructions in a cue-target memory task (Dobbins and Han, 2006). Activity in VLPFC is not commonly observed during attentional cueing (Corbetta et al., 2008). In the memory domain, activity in VLPFC has been linked with the selection of taskrelevant memories among distractors (Badre and Wagner, 2007). This process was arguably necessary in our task to guide memory search to the retrieval of the original associates of relevant cues (Kuhl et al., 2007), but less crucial in perceptual-attentional tasks where cue-target relations are not ambiguous.

\section{Bottom-up AtoM}

Two findings support a role for VPC in bottom-up AtoM. First, a region in left VPC located between the angular gyrus and the middle temporal gyrus $\left(\mathrm{VPC}_{\mathrm{AnG} / \mathrm{MTG}}\right)$ predicted efficacy and speed of target detection when no cue was provided, and therefore the recognition decisions had to rely mainly on bottom-up signals originating from the memory probe. In contrast, $\mathrm{VPC}_{\mathrm{AnG} / \mathrm{MTG}}$ was not responsive to memory cueing. These response properties, similar to those exhibited by right VPC in the perceptualattentional domain (Corbetta et al., 2000), suggest that VPC mediates the automatic attentional capture by recollected memories. In line with this interpretation, $\mathrm{VPC}_{\mathrm{AnG} / \mathrm{MTG}}$ is close to $\mathrm{VPC}$ sites activated by recognition decisions accompanied by high confidence (median coordinates: $x=-48, y=-63, z=25$ ) (Cabeza et al., 2008), which usually occurs when a large amount of information is rapidly retrieved from memory that captures attention bottom up.

Moreover, the left angular gyrus $\left(\mathrm{VPC}_{\mathrm{AnG}}\right)$ responded more intensely when mnemonic expectations were violated (Recombined condition) than when they were met (Intact condition), or not formed at all (NoOld and NewOld conditions). Studies in the perceptual-attentional domain have shown that VPC regions, including the angular gyrus, mediate reorienting of attention after invalid cueing (Arrington et al., 2000; Kincade et al., 2005; Indovina and Macaluso, 2007), thus enabling detection of relevant, yet unattended stimuli (Corbetta et al., 2008). Accordingly, patients with neglect due to right VPC lesions fail to detect targets in the contralesional hemifield, especially after invalid cueing (Friedrich et al., 1998). Our results suggest that left VPC is implicated in detecting relevant yet unexpected memory contents. In real life, this process is likely engaged when a memory pops out spontaneously that interrupts pre-existing mental sets (e.g., "Wait, I have to see the dentist today!"). Left VPC may be necessary to mediate a switch signal (Corbetta et al., 2008) that disengages attention from the ongoing focus of processing (Posner et al., 1984) and reorients it toward incoming memory contents so that these can gain access to awareness. Consistent with this hypothesis, VPC patients had problems with recognizing unexpected memory targets (Recombined condition) despite not being generally impaired in recognition memory. Notably, the increased susceptibility of VPC patients to invalid memory cueing in the Recombined condition indicates that VPC is not necessary to encode or retrieve associative information. A selective deficit in bottom-up AtoM mechanisms in the face of generally preserved memory processes can explain why VPC patients are disproportionately impaired in spontaneous, compared with prompted, memory retrieval (Berryhill et al., 2007; Simons et al., 2008) (but see Davidson et al., 2008).

In addition to VPC, activity in left lateral anterior PFC and DLPFC accompanied detection of targets for memory. Left lateral anterior PFC has long been implicated in the successful detection (recognition) of studied items (Rugg and Henson, 2002). Activity in this region is modulated according to familiarity confidence (Yonelinas et al., 2005; Montaldi et al., 2006) and is larger when memory targets are rare compared with frequent (Herron et al., 2004), consistent with a role in the bottom-up attentional capture by behaviorally salient memory contents. Memory detection was also accompanied by activity in left DLPFC. Different from VPC, however, DLPFC mediated slow recognition responses, suggesting this region was not implicated in recognition of memory targets that captured attention, but rather of targets that were difficult to identify.

One further aspect of our findings merits discussion. As Figure 7 shows, we observed a graded difference and not sharp dissociations in the involvement of VPC and DPC in AtoM. Although DPC and VPC exhibited a preferential response to effects related to top-down and bottom-up AtoM, respectively, the overall pattern of responding in these regions was not dramatically different from a qualitative point of view. This finding likely reflects the fact that VPC and DPC interact closely during memory retrieval, as they do in attention (Cabeza et al., 2008; Corbetta et al., 2008). Because retrieval goals are constantly updated in DPC, DPC may be indirectly sensitive to target detection. As well, a change in retrieval goals updates the saliency of memory contents and may influence VPC activity related to detection. Our finding that lesions to DPC and VPC caused qualitatively different impairments, however, indicates that DPC is crucial for topdown AtoM, whereas VPC is crucial for bottom-up AtoM.

AtoM regions were partially segregated from regions associated with retrieval success effects. We propose that AtoM regions may take part in neural mechanisms specialized to provide attentional support to memory and nonmemory tasks and are recruited during memory retrieval only under conditions that pose demands on bottom-up and top-down AtoM. Although speculative at this point, this proposal is in line with the finding from the current study, as well as other studies (Berryhill et al., 2007; Davidson et al., 2008), that PPC patients may show deficits in top-down and bottom-up AtoM but not a generalized memory impairment. Subtle memory deficits, related to AtoM problems, are likely to go undetected in clinical practice (Luria, 1976).

Although our results support the AtoM hypothesis, alternative interpretations of the role of the PPC in memory retrieval must be considered. According to the mnemonic accumulator hypothesis (Wagner et al., 2005), PPC integrates information coming from other brain regions in a signal that determines the recognition response. That activity in VPC predicts hit rates is consistent with an accumulator role. We note, however, that previous research has emphasized the role of intraparietal, not ventral parietal, regions in information accumulation in both memory (Yago and Ishai, 2006; Ploran et al., 2007) and nonmemory domains (Colby and Goldberg, 1999; Platt and Glimcher, 1999). Besides, the 
mnemonic accumulator hypothesis would not predict special sensitivity to unexpected targets.

According to the output buffer hypothesis (Vilberg and Rugg, 2007, 2008), VPC helps hold retrieved contents in an episodic buffer. This hypothesis leads to the prediction of stronger activity when a large amount of information is revived in the buffer. Our finding that activity in VPC increased when mnemonic expectations were violated, while not merely tracking the amount of retrieved information, is more consistent with an attentional account of VPC activity.

Finally, PPC may index the magnitude of the subjective episodic experience occurring at recognition (Ally et al., 2008). This hypothesis does not make differential predictions for DPC and VPC and thus cannot fully explain our data. However, assessing subjective correlates of retrieval may help elucidate the role of PPC in episodic memory.

One criticism of the AtoM hypothesis involves the distribution of activation in episodic retrieval and attention studies (for discussion, Hutchinson et al., 2009). The IPS sites involved in top-down AtoM are slightly lateral to those implicated in attentional cueing. As for bottom-up AtoM, whereas detection of memory targets is associated with left VPC activity, target detection in "Posner" perceptual-attentional paradigms is, with a few exceptions (Kincade et al., 2005; Indovina and Macaluso, 2007), lateralized to the right hemisphere (Corbetta et al., 2000). It is very likely that PPC sites supporting top-down and bottom-up attention depend, at least in part, on the type of predictive cue (Nobre, 2004; Cristescu et al., 2006) and on whether attention is directed externally or internally (Corbetta et al., 2008; Uncapher and Wagner, 2009). For example, studies investigating the ability to orient attention to semantic categories (Cristescu et al., 2006) found activity in left IPS regions close to ours. Further research will be needed to clarify the role of the domain (e.g., perception, memory, action) to which attention is applied in determining the organization and lateralization of different regions of parietal cortex.

In summary, we report, in a single study, neuroimaging and lesion evidence consistent with the AtoM hypothesis: DPC is necessary to search for relevant memories, whereas VPC is necessary to detect memory contents rapidly, especially when they compete with pre-existing goals.

\section{References}

Ally BA, Simons JS, McKeever JD, Peers PV, Budson AE (2008) Parietal contributions to recollection: electrophysiological evidence from aging and patients with parietal lesions. Neuropsychologia 46:1800-1812.

Arrington CM, Carr TH, Mayer AR, Rao SM (2000) Neural mechanisms of visual attention: object-based selection of a region in space. J Cogn Neurosci 12:106-117.

Badre D, Wagner AD (2007) Left ventrolateral prefrontal cortex and the cognitive control of memory. Neuropsychologia 45:2883-2901.

Barca L, Burani C, Arduino LS (2002) Word naming times and psycholinguistic norms for Italian nouns. Behav Res Methods Instrum Comput 34:424-434.

Berryhill ME, Phuong L, Picasso L, Cabeza R, Olson IR (2007) Parietal lobe and episodic memory: bilateral damage causes impaired free recall of autobiographical memory. J Neurosci 27:14415-14423.

Cabeza R (2008) Role of parietal regions in episodic memory retrieval: the dual attentional processes hypothesis. Neuropsychologia 46:1813-1827.

Cabeza R, Ciaramelli E, Olson I, Moscovitch M (2008) The parietal cortex and episodic memory: an attentional account. Nat Rev Neurosci 9:613-625.

Ciaramelli E, Grady C, Moscovitch M (2008) Top-down and bottom-up attention to memory: a hypothesis (AtoM) on the role of the posterior parietal cortex in memory retrieval. Neuropsychologia 46:1828-1851.
Colby CL, Goldberg ME (1999) Space and attention in parietal cortex. Annu Rev Neurosci 22:319-349.

Corbetta M, Shulman GL (2002) Control of goal-directed and stimulusdriven attention in the brain. Nat Rev Neurosci 3:201-215.

Corbetta M, Kincade JM, Ollinger JM, McAvoy MP, Shulman GL (2000) Voluntary orienting is dissociated from target detection in human posterior parietal cortex. Nat Neurosci 3:292-297.

Corbetta M, Patel G, Shulman G (2008) The reorienting system of the human brain: from environment to theory of mind. Neuron 58:306-324.

Cox RW (1996) AFNI: software for analysis and visualization of functional magnetic resonance neuroimages. Comput Biomed Res 29:162-173.

Crawford JR, Garthwaite PH (2002) Investigation of the single case in neuropsychology: confidence limits on the abnormality of test scores and test score differences. Neuropsychologia 40:1196-1208.

Cristescu TC, Devlin JT, Nobre AC (2006) Orienting attention to semantic categories. Neuroimage 33:1178-1187.

Davidson PS, Anaki D, Ciaramelli E, Cohn M, Kim AS, Murphy KJ, Troyer AK, Moscovitch M, Levine B (2008) Does lateral parietal cortex support episodic memory? Evidence from focal lesion patients. Neuropsychologia 46:1743-1755.

Dobbins IG, Han S (2006) Cue- versus probe-dependent prefrontal cortex activity during contextual remembering. J Cogn Neurosci 18:1439-1452.

Fisher RA (1950) Statistical methods for research workers. London: Oliver and Boyd.

Fleck MS, Daselaar SM, Dobbins IG, Cabeza R (2006) Role of prefrontal and anterior cingulate regions in decision-making processes shared by memory and nonmemory tasks. Cereb Cortex 16:1623-1630.

Friedrich FJ, Egly R, Rafal RD, Beck D (1998) Spatial attention deficits in humans: a comparison of superior parietal and temporal-parietal junction lesions. Neuropsychology 12:193-207.

Henson RN, Rugg MD, Shallice T, Dolan RJ (2000) Confidence in recognition memory for words: dissociating right prefrontal roles in episodic retrieval. J Cogn Neurosci 12:913-923.

Herron JE, Henson RN, Rugg MD (2004) Probability effects on the neural correlates of retrieval success: an fMRI study. Neuroimage 21:302-310.

Hutchinson JB, Uncapher MR, Wagner AD (2009) Posterior parietal cortex and episodic retrieval: convergent and divergent effects of attention and memory. Learn Mem 16:343-356.

Indovina I, Macaluso E (2007) Dissociation of stimulus relevance and saliency factors during shifts of visuospatial attention. Cereb Cortex 17:1701-1711.

Kim H, Cabeza R (2007) Trusting our memories: dissociating the neural correlates of confidence in veridical versus illusory memories. J Neurosci 27:12190-12197.

Kincade JM, Abrams RA, Astafiev SV, Shulman GL, Corbetta M (2005) An event-related functional magnetic resonance imaging study of voluntary and stimulus-driven orienting of attention. J Neurosci 25:4593-4604.

Konishi S, Wheeler ME, Donaldson DI, Buckner RL (2000) Neural correlates of episodic retrieval success. Neuroimage 12:276-286.

Kucera H, Francis WN (1967) Computational analysis of present-day American English. Providence: Brown UP.

Kuhl BA, Dudukovic NM, Kahn I, Wagner AD (2007) Decreased demands on cognitive control reveal the neural processing benefits of forgetting. Nat Neurosci 10:908-914.

Lazar NA, Luna B, Sweeney JA, Eddy WF (2002) Combining brains: a survey of methods for statistical pooling of information. Neuroimage 16:538-550.

Luria AR (1976) The neuropsychology of memory. Oxford: Winston.

Marois R, Leung HC, Gore JC (2000) A stimulus-driven approach to object identity and location processing in the human brain. Neuron 25:717-728.

McDermott KB, Jones TC, Petersen SE, Lageman SK, Roediger HL 3rd (2000) Retrieval success is accompanied by enhanced activation in anterior prefrontal cortex during recognition memory: an event-related fMRI study. J Cogn Neurosci 12:965-976.

Miller EK, Cohen JD (2001) An integrative theory of prefrontal cortex function. Annu Rev Neurosci 24:167-202.

Montaldi D, Spencer TJ, Roberts N, Mayes AR (2006) The neural system that mediates familiarity memory. Hippocampus 16:504-520.

Nobre AC (2004) Probing the flexibility of attentional orienting in the human brain. In: Cognitive neuroscience of attention (Posner MI, ed). New York: Guilford. 
Platt ML, Glimcher PW (1999) Neural correlates of decision variables in parietal cortex. Nature 400:233-238.

Ploran EJ, Nelson SM, Velanova K, Donaldson DI, Petersen SE, Wheeler ME (2007) Evidence accumulation and the moment of recognition: dissociating perceptual recognition processes using fMRI. J Neurosci 27:11912-11924.

Posner MI, Petersen SE (1990) The attention system of the human brain. Annu Rev Neurosci 13:25-42.

Posner MI, Walker JA, Friedrich FJ, Rafal RD (1984) Effects of parietal injury on covert orienting of attention. J Neurosci 4:1863-1874.

Rorden C, Brett M (2000) Stereotaxic display of brain lesions. Behav Neurol 12:191-200.

Rugg MD, Henson RNA (2002) Episodic memory retrieval: an (eventrelated) functional neuroimaging perspective. In: The cognitive neuroscience of memory encoding and retrieval (Parker AE, Wilding EL, Bussey T, eds). New York: Psychology.

Shulman GL, Astafiev SV, Franke D, Pope DL, Snyder AZ, McAvoy MP, Corbetta M (2009) Interaction of stimulus-driven reorienting and expectation in ventral and dorsal frontoparietal and basal ganglia-cortical networks. J Neurosci 29:4392-4407.

Simons JS, Peers PV, Hwang DY, Ally BA, Fletcher PC, Budson AE (2008) Is the parietal lobe necessary for recollection in humans? Neuropsychologia 46:1185-1191.

Talairach J, Tournoux P (1988) Co-planar stereotaxic atlas of the human brain. New York: Thieme.

Uncapher MR, Rugg MD (2009) Selecting for memory? The influence of selective attention on the mnemonic binding of contextual information. J Neurosci 29:8270-8279.

Uncapher MR, Wagner AD (2009) Posterior parietal cortex and episodic encoding: Insights from fMRI subsequent memory effects and dualattention theory. Neurobiol Learn Mem 91:139-154.

Vilberg KL, Rugg MD (2007) Dissociation of the neural correlates of recognition memory according to familiarity, recollection, and amount of recollected information. Neuropsychologia 45:2216-2225.

Vilberg K, Rugg MD (2008) Memory retrieval and the parietal cortex: a review of evidence from a dual-process perspective. Neuropsychologia 46:1787-1799.

Wagner AD, Shannon BJ, Kahn I, Buckner RL (2005) Parietal lobe contributions to episodic memory retrieval. Trends Cogn Sci 9:445-453.

Wheeler ME, Buckner RL (2003) Functional dissociation among components of remembering: control, perceived oldness, and content. J Neurosci 23:3369-3880.

Wheeler ME, Shulman GL, Buckner RL, Miezin FM, Velanova K, Petersen SE (2006) Evidence for separate perceptual reactivation and search processes during remembering. Cereb Cortex 16:949-959.

Yago E, Ishai A (2006) Recognition memory is modulated by visual similarity. Neuroimage 31:807-817.

Yonelinas AP, Otten LJ, Shaw KN, Rugg MD (2005) Separating the brain regions involved in recollection and familiarity in recognition memory. J Neurosci 25:3002-3008. 